\title{
A Chipless RFID Method of 2D Localization Based on Phase Acquisition
}

\author{
Nicolas Barbot ${ }^{1}{ }^{1}$ and Etienne Perret ${ }^{1,2}$ \\ ${ }^{1}$ Univ. Grenoble Alpes, Grenoble INP, LCIS, 26000 Valence, France \\ ${ }^{2}$ Institut Universitaire de France, Paris, France \\ Correspondence should be addressed to Nicolas Barbot; nicolas.barbot@lcis.grenoble-inp.fr
}

Received 16 February 2018; Revised 26 April 2018; Accepted 3 June 2018; Published 19 July 2018

Academic Editor: Oleg Lupan

Copyright (c) 2018 Nicolas Barbot and Etienne Perret. This is an open access article distributed under the Creative Commons Attribution License, which permits unrestricted use, distribution, and reproduction in any medium, provided the original work is properly cited.

\begin{abstract}
This paper explores the performance of object localization using chipless tags. We show that it is possible to localize a tag (or an object attached to it) by measuring the phase offset between a known position and the position to estimate. This method provides better accuracy compared to classical ones based on received signal strength indicator (RSSI) or round-trip time of flight. We show that submillimeter precision for distance measurement and an error of less than $4 \mathrm{~mm}$ for localization can be achieved. These results point the way toward new kinds of sensors and user interfaces using chipless tags which can be contactless and 3D. This new possibility is in addition to the identification functionality which is inherent to the use of chipless tags.
\end{abstract}

\section{Introduction}

RFID technology is a mature technology to identify items. A single \$0.3 RFID chip contains a 96-bit electronic product code used to uniquely identify a tag. However, classical RFID technology suffers from its cost which is too expensive for a large number of applications. Chipless RFID technology offers an alternative and a complementary solution to classical RFID and can permit to drastically decrease tag costs. In chipless RFID, tags do not include any chip to store information, nor to modulate the backscattered signal. Instead, the information is directly encoded inside the tag structure. It is why in terms of targeted applications, chipless RFID is closer to the barcode technology rather than to the classical RFID. Compared to barcodes, chipless tags offer better discretion (since line of sight is not required), higher read range (up to $50 \mathrm{~cm}$ ), and a better robustness against misalignment or misorientation [1]. Table 1 presents a comparison between classical RFID, chipless RFID, and barcode.

A lot of effort has already been made to increase the coding capacity and the robustness of the reading [1]. With this technology, tag cost can be reduced to 0.4 cents [2] and can bring, for almost the same cost, additional services compared to barcodes.

In this paper, we focus on adding new functionalities to chipless tags. Sensing physical quantities (temperature, material permittivity, location, ...) is one of the most promising capabilities. Localization of a chipless tag simply using the tag response can enable the development of new kinds of user interfaces which can be contactless and 3D. The objective of this paper is to localize an object on a 2D plan using a chipless tag with high accuracy. Since the tag is attached to the object, the problem is equivalent to find the position of a chipless tag on a $2 \mathrm{D}$ plan. Localization using chipless tags has already been investigated in the literature. In [3-5], the authors have realized localization by using round-trip time-of-flight and trilateration algorithms to recover the position of a tag. These approaches use at least 3 different antennas and have been focused on the structural mode of the tag (i.e., the early part response of the backscattered wave) to determine the distance information. In [6], the author has used the phase of the reflected signal to measure distance variation with submillimeter accuracy along a single direction. Moreover, in [7], the authors have determined the localization of a chipless 
TABLE 1: Comparison between classical RFID, chipless RFID, and barcode.

\begin{tabular}{lccc}
\hline & RFID UHF & Chipless RFID & Barcode \\
\hline Reliability & Very good & Good & Very good \\
Multiread & Possible & Difficult & Impossible \\
Read range & $10 \mathrm{~m}$ & $50 \mathrm{~cm}$ & $50 \mathrm{~cm}$ \\
Discretion & Nonline & Nonline & Line \\
Cost & of sight & of sight & of sight \\
Rewritable & $\$ 0.4$ & $\$ 0.004$ & $<\$ 0.0001$ \\
Sensor & Possible & Possible & Impossible \\
Gesture recognition & Impossible & Possible & Impossible \\
\hline
\end{tabular}

tag using the change of the magnitude of the backscattered signal using the information of each of the tag's scatterer. This latter method provides an absolute positioning but suffers from a relatively low accuracy.

In this paper, we propose a new method to localize a chipless tag on a $2 \mathrm{D}$ plan. This method uses a single reader antenna and relies on the phase measurement of the antenna mode of the tag to estimate the distance between a fixed point and all resonators. This information is then used to precisely localize the tag with a multilateration technique.

Last but not least, this sensing functionality does not come at a price of a loss in the coding capacity for the identification functionality (the tag can still be used to identify an item with the same coding capacity). Thus, the results presented in this paper could be applied to any tag operating in a frequency domain.

Chipless RFID principles and the analytical model used to localize a tag are presented in Section 2. Results, including measurement bench and performance evaluation, are presented in Section 3 before concluding.

\section{Concept of Phase Measurement for Chipless Tag Localization}

2.1. Chipless RFID Technology. Chipless tags operating in the frequency domain are classically composed by multiple resonators [8]. Each resonator is a simple structure, where the resonant frequency mainly depends on a parameter (typically its length). The combination of multiple resonant frequencies can be used to identify a chipless tag [1]. Each resonator backscatters power preferentially around its resonant frequency; so, in order to determine the characteristics of a given tag, a reader has to realize two different operations: (1) on the emitter side, the reader has to transmit power toward the tag (pulses or harmonic sweep) and (2) on the receiving side, the reader has to estimate the magnitude of the received signal as a function of the frequency to identify the resonant frequencies. However, even if the magnitude of the received signal is sufficient to identify a tag, it does not contain all the information of the backscattered signal. Many characteristics are also included in the phase information. In the proposed method, the phase of the backscatter signal is exploited to obtain information on the location of a chipless tag.

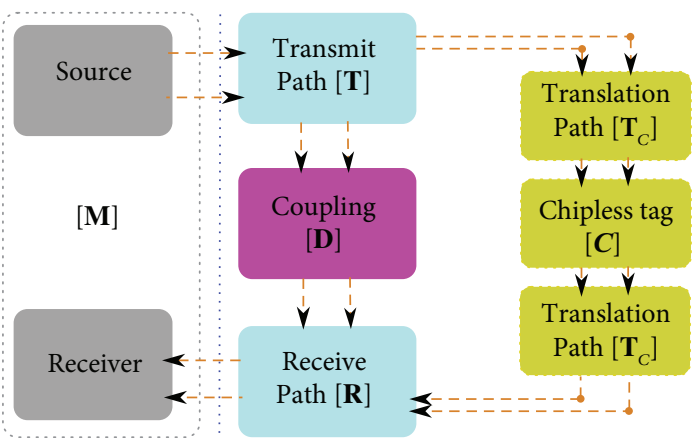

Figure 1: Channel model of the system, accounting for displacement measurements with one chipless tag.

2.2. Analytical Model. Figure 1 presents the channel model of the transmission between the reader and the chipless tag [9]. The reader acting as a detection system is represented by block $[\mathbf{M}]$. Blocks $[\mathbf{T}]$ and $[\mathbf{R}]$ represent the transmitting and the receiving paths, respectively. Coupling between emitting and receiving antennas is noted as $[\mathbf{D}]$, and block $[\mathbf{C}]$ represents the chipless tag. Each block is a matrix of the form

$$
[\mathbf{X}]=\left[\begin{array}{ll}
X_{v v} & X_{v h} \\
X_{h v} & X_{h h}
\end{array}\right] .
$$

Since the tag can be located at a given distance from the antenna, block $\left[\mathbf{T}_{C}\right]$ represents the phase offset due to propagation [6]:

$$
\left[\mathbf{T}_{C}\right]=\left[\begin{array}{cc}
e^{-j k_{0} d} & 0 \\
0 & e^{-j k_{0} d}
\end{array}\right],
$$

where $d$ is the distance between the tag and the antenna and $k_{0}=2 \pi / \lambda$. From this model, one can extract the signal received in cross-polarization at a known reference position $d_{0}$ and at an unknown distance $d_{1}$ as [6]

$$
\begin{aligned}
& \mathbf{M}_{v h}^{d_{0}}=I_{v h}+\mathbf{T}_{h h} \mathbf{C}_{v h} e^{-j 2 k d_{0}} \mathbf{R}_{v v}, \\
& \mathbf{M}_{v h}^{d_{1}}=I_{v h}+\mathbf{T}_{h h} \mathbf{C}_{v h} e^{-j 2 k d_{1}} \mathbf{R}_{v v},
\end{aligned}
$$

where $I_{v h}$ is the direct coupling between antennas, also called isolation measurement $\left(M_{v h}^{d_{0}}=I_{v h}\right.$ if there is no tag $[\mathbf{C}]$ in the front of the antenna). Symbols $h$ and $v$ denotethe power waves in the horizontal $(h)$ and vertical $(v)$ polarizations, respectively. From [6], a distance variation can be extracted by using only 2 measurements $\left(M_{v h}^{d_{0}}\right.$ and $\left.M_{v h}^{d_{1}}\right)$ at 2 different locations $d_{0}$ and $d_{1}$ and a background measurement $I_{v h}$. Distance variation $d_{1}-d_{0}$ can thus be extracted from (2) by subtracting $I_{v h}$ in both equations and by dividing the second equation by the first one. The following result can be obtained:

$$
\left(d_{1}-d_{0}\right)_{(i)}=-\frac{1}{2 k} \text { angle }\left(\frac{\mathbf{M}_{v h}^{d_{1}}\left(f_{i}\right)-I_{v h}\left(f_{i}\right)}{\boldsymbol{M}_{v h}^{d_{0}}\left(f_{i}\right)-I_{v h}\left(f_{i}\right)}\right) .
$$




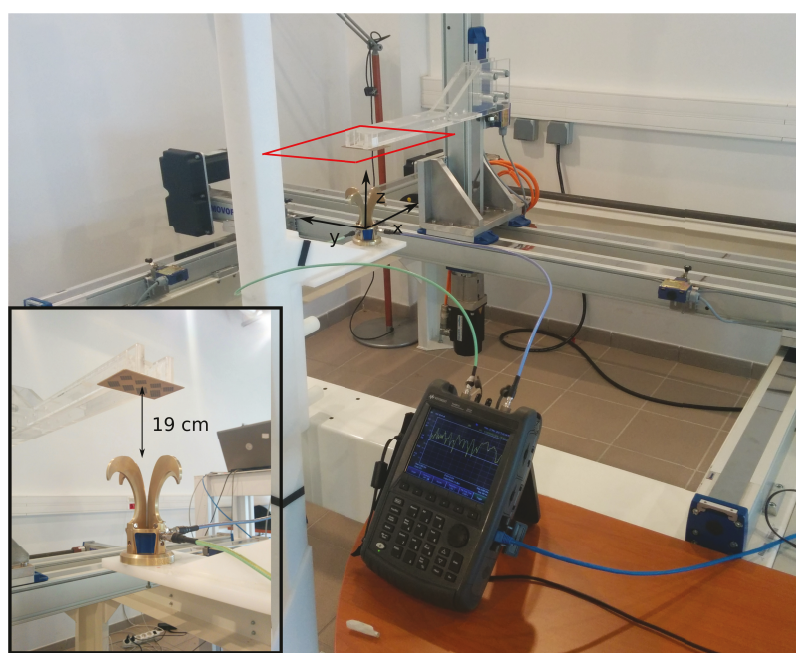

FIgURE 2: Measurement bench in a real environment for $2 \mathrm{D}$ localization.

Note that (3) holds in cross-polarization and only at the neighbourhood of the resonant frequency since at other frequencies, power reflected by other objects surrounding the reader can be higher than the power backscattered by the $\operatorname{tag}$ [6]. Moreover if one distance is known (e.g., $d_{0}$ ), absolute distance $d_{1}$ can thus be determined. In the following, this position at which the distance is known is called the reference position. Finally, if the tag contains more than one resonator, distances between each resonator and the reference position can also be determined independently. The following section describes the measurement bench used to localize a chipless tag.

\section{Experimental Validation and Discussion}

3.1. Measurement Bench. In order to validate the model, the measurement bench has been set in a real environment as presented in Figure 2. Tag response has been captured using a 2-port antenna (cross-polarization measurement). This dual-access dual-polarization Satimo QH2000 antenna is connected to the VNA ports 1 and 2 in the horizontal and vertical polarizations, respectively. The tag's reference position $(0,0)$ has been taken when the tag is placed at broadside relative to the antenna. The distance between the tag and the antenna is $19 \mathrm{~cm}$ (see Figure 2). Then, the tag is moved on a horizontal square of $20 \mathrm{~cm}$ side length (red square in Figure 2), centered on the reference position, with a displacement step of $1 \mathrm{~cm}$ on both axes. For each position, 10 measurements between $2.8 \mathrm{GHz}$ and $8 \mathrm{GHz}$ with 201 points are recorded. In the study, we do not consider displacement of external objects.

The chipless tag chosen for this study is a crosspolarization tag composed of 8 resonators. In [9], the authors have shown that this tag has a coding capacity of 19 bits. Classical response in magnitude of this tag is presented in Figure 3. This response has been obtained after removing the environment response $I_{v h}$ (i.e., the same measurement but without the tag) and without using time gating. The eight resonant frequencies, corresponding to the eight resonators,

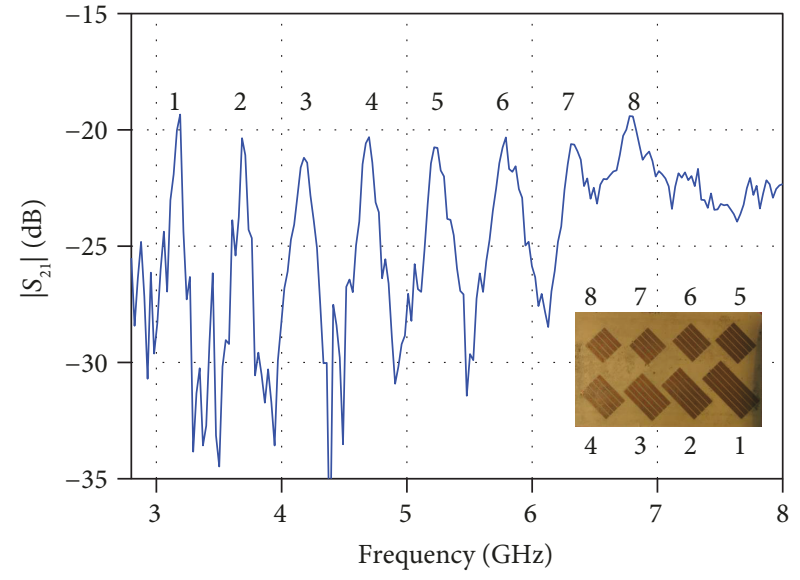

Figure 3: Response of the measured tag in magnitude at the reference position $(0,0)$ using the measurement bench presented in Figure 2.

are clearly visible on the magnitude response. Shorter resonators correspond to higher resonant frequencies. The location of these peaks is used to realize the identification function of the tag.

In the previous section, it has been shown that it is possible to determine theoretically the distance variations between two positions or the absolute distance from the antenna if a known reference position is used. In this part, the phase at the resonant frequency of the first resonator $\left(n^{\circ} 1\right.$ in Figure 3, resonating at $3.19 \mathrm{GHz})$ and last resonator $\left(n^{\circ} 8\right.$ in Figure 3, resonating at $6.78 \mathrm{GHz}$ ) of the received signal is determined for each position ( $x$ and $y$ ). The obtained results are shown in Figure 4. For comparative purposes, the plot has been also done for frequencies outside the peak apex (3.39 GHz and 7.70 GHz). For the two resonant frequencies, the phase exhibits concentric patterns. Moreover, the pattern center corresponds to the position where the given resonator is at the closest distance to the antenna (these positions are different because resonators are not located exactly at the same place, as seen in Figure 4). On the other side, the phase at nonresonant frequencies presents unpredictable behaviour (but remains deterministic).

3.2. Distance Determination. From this result, we can now estimate the distance variations between the reference position and any other position into the $2 \mathrm{D}$ plane. To apply (3), the phase needs first to be unwrapped by adding multiples of $\pm 2 \pi$ to compensate the variations observed in Figures 4(a) and 4(b). This unwrapping is a complex operation since it has to be done jointly along the $x$ and $y$ axes. In this paper, we have used the algorithm proposed in [10]. 2D phase unwrapping is a critical operation for distance determination and localization and will directly impact the performance of our localization method when we consider distance variation larger than one wavelength. In Figures 4(e) and 4(f), errors during unwrapping are clearly visible in the upper-left corner and upper-right corner, respectively. Absolute distance for each resonator can thus be estimated by adding vectorially the distance of the 


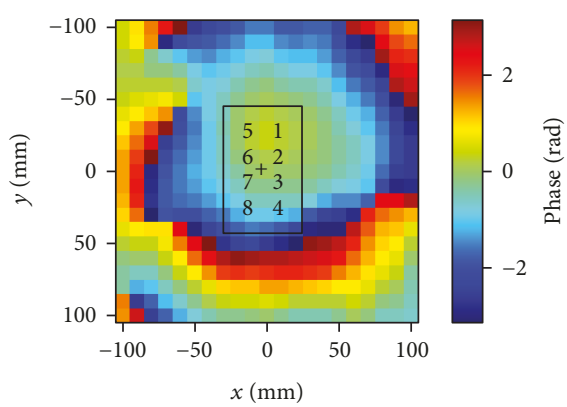

(a)

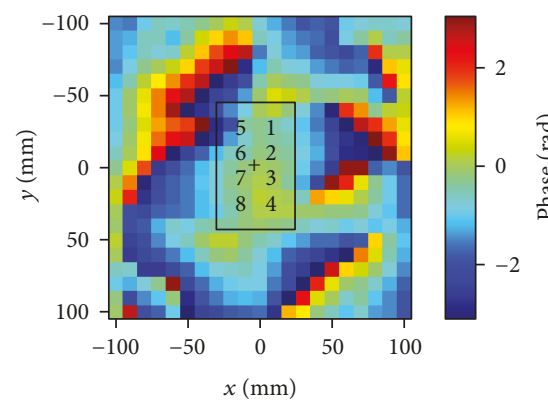

(d)

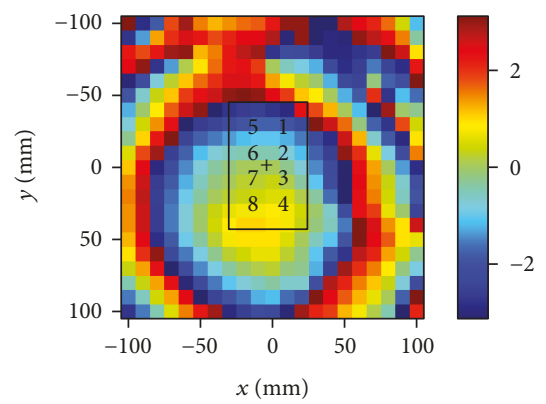

(b)

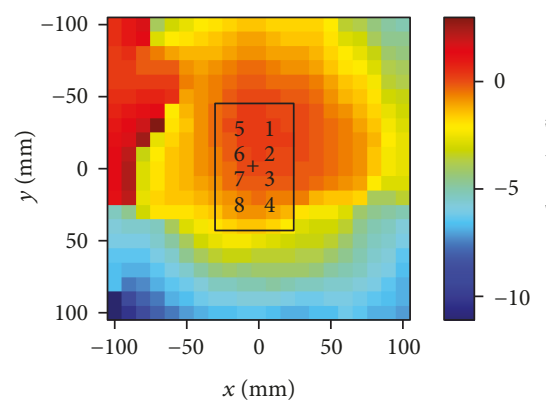

(e)

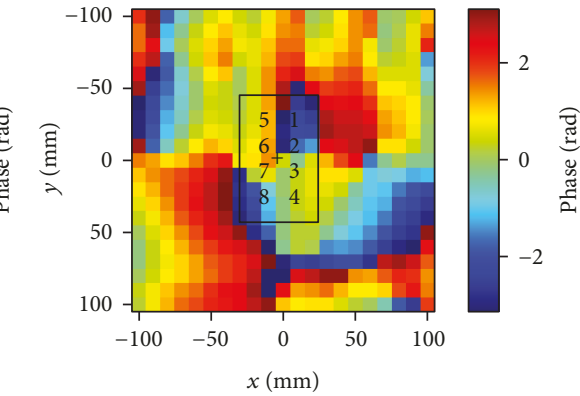

(c)

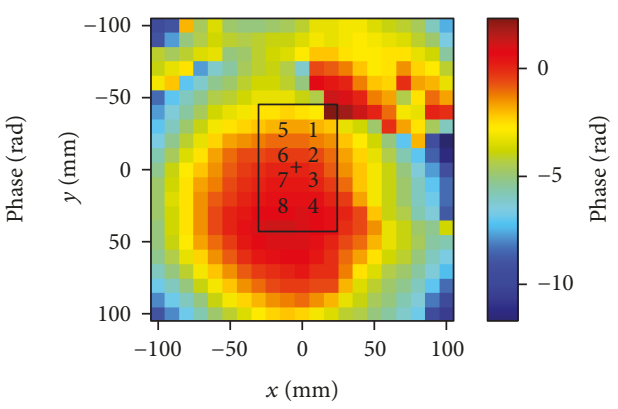

(f)

Figure 4: Phase variation as a function of the position on the plan, (a) for the first resonant frequency (3.19 GHz), (b) for the 8th resonant frequency $(6.78 \mathrm{GHz}),(\mathrm{c})$ at a frequency located between the first and the second peaks $(3.39 \mathrm{GHz}),(\mathrm{d})$ at a frequency located after the 8 th peak $(7.70 \mathrm{GHz})$, (e) on the phase unwrapped for the first resonant frequency $(3.19 \mathrm{GHz})$, and (f) on the phase unwrapped for the 8 th resonant frequency $(6.78 \mathrm{GHz})$.

reference position to the distance variations extracted by phase measurements. Figure 5 presents the absolute distance between resonators $1,2,3$, and 4 and the antenna when the tag is moved along the $x$ and $y$ axes form 0 to $50 \mathrm{~mm}$. Theoretical distance is also presented on the same plot.

In Figure 5(a), the two lower curves correspond to the inner resonators $\left(\mathrm{n}^{\circ} 2,3\right)$ which are closer and located at an equal distance from the antenna. The two upper curves correspond to the two outer resonators $\left(n^{\circ} 1,4\right)$. We can also see that the distance predicted with (3) is very close to the theoretical distance. The same results can be observed for the $y$ axis (see Figure 5(b)). To estimate the accuracy of our distance measurement, the variance of the error between different measurements for the same location has been computed. This variance for all resonators is less than $20 \mu \mathrm{m}$ (this result does not take into account errors due to the tag's position).

3.3. Localization Performance. Since distance measurement has been validated, these results can be used to estimate the location of the chipless tag on a $2 \mathrm{D}$ plan located at $19 \mathrm{~cm}$ from the antenna. As the relative positions between resonators are known (see Figure 3) and distances between each resonator and the antenna can be extracted as previously shown, it is possible to localize the tag using multilateration algorithms. Different solutions have been developed in [11, 12] but remain relatively complex. In the following, a heuristic algorithm is considered to recover the $x$ and $y$ coordinates of the tag in the 2D plane. These coordinates are directly extracted from the distances between the reference position and the unknown position by using a simple random search. This algorithm determines the coordinates $(x, y)$ of the tag by minimizing the mean square error (MSE) between the 8 distances obtained with the phase measurement and a candidate position. At the beginning of the procedure, this candidate position is initialized at the reference position. For each iteration, a small perturbation along $x$ and $y$ is added to the candidate position and the MSE is computed. If the MSE value is lower than the one computed at the previous iteration, the candidate position is updated to the new position. Thus, this search iteratively converges to the tag position. Compared to classical methods (like Gauss-Newton algorithm), this search does not require any information from the objective function and is less sensitive to noise. Location estimation has been computed for every position and distance error has been estimated over the entire plan. Figure 6 presents the localization error as a function of $x$ and $y$.

We can see that localization error remains low over a wide portion of the 2D plan. However, for some regions, the localization exhibits a large error (more than $50 \mathrm{~mm}$ ). This behaviour is due to the phase unwrapping process which wrongly modifies the phase (see Figures $4(\mathrm{e})$ and $4(\mathrm{f})$ ) when the tag is no more localized in the antenna's mean beam. Indeed, as the $2 \mathrm{D}$ plan is relatively close to the antenna and due to the high antenna directivity, low signal is sent form directions others than the mean beam. This error thus degrades the performance of the multilateration method. This explains the concentric shape observed in Figure 6, as well as the fact that the distance errors drastically increase when the tag is shifted by $8 \mathrm{~cm}$ from the center. Note that 


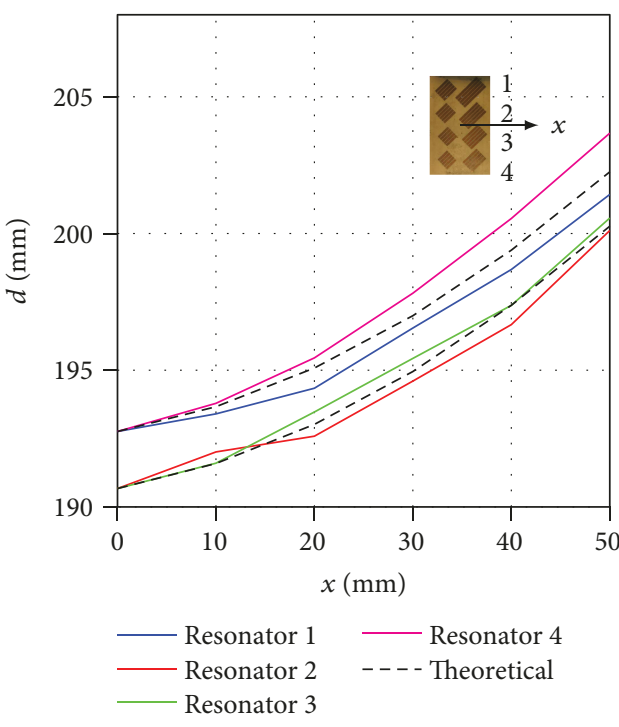

(a)

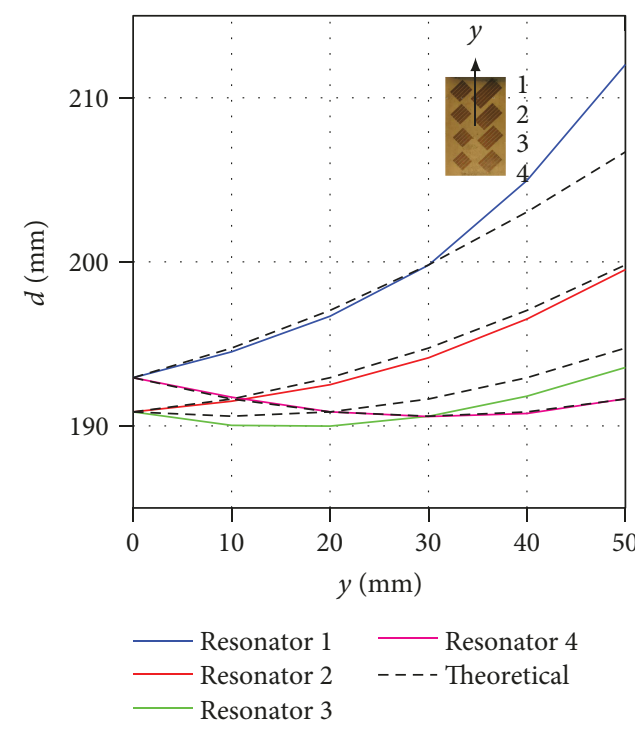

(b)

Figure 5: Distance estimation for 4 resonators ( $n^{\circ} 1$ to $\left.n^{\circ} 4\right)$ (plain) and theoretical distance (dashed) as a function of (a) $x$ and (b) $y$.

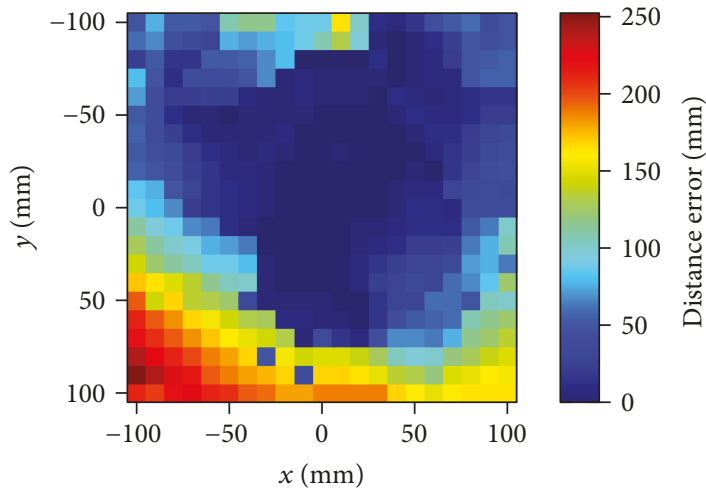

Figure 6: Localization error as a function of $x$ and $y$.

inside this $8 \mathrm{~cm}$ radius, the distance error tends to be 0 . In order to avoid this type of errors, a subset of the $2 \mathrm{D}$ plan formed by an $80 \mathrm{~mm}$ side square centered on the reference position is considered. Figure 7 presents this study where some chosen positions around the reference position and the corresponding coordinates estimated by our method are plotted. For each position, 10 coordinates are determined corresponding to the 10 measurements. Estimated positions are very close to the theoretical ones.

From these results, position error can be determined to evaluate the performance of this localization technique. Results in term of error along the $x$ and $y$ axes are presented in Figure 8.

It can be seen that the error on both axes is centered at 0 and is less than $\pm 7 \mathrm{~mm}$. Mean and standard deviation for the $x$ error are equal to $\mu_{x}=-0.7 \mathrm{~mm}$ and $\sigma_{x}=3.5 \mathrm{~mm}$, respectively (and $\mu_{y}=-0.2 \mathrm{~mm}$ and $\sigma_{y}=2.0 \mathrm{~mm}$ for the axis, resp.). Average error distance can thus be extracted from these results and is equal to $3.58 \mathrm{~mm}$. Thus, the accuracy of our proposed method is high enough to be used in

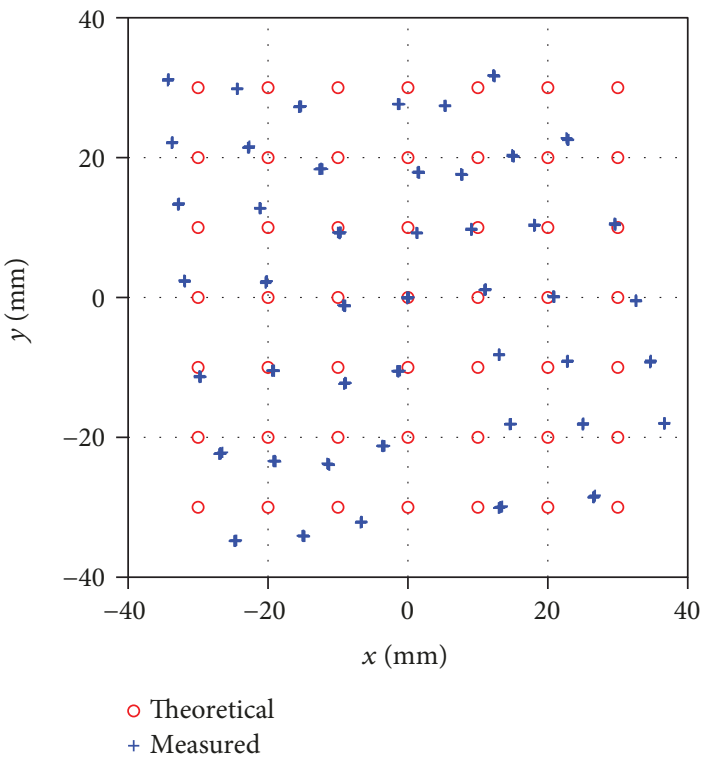

FIgURE 7: Tag positons obtained with the proposed method (blue cross) compared to the expected positions (red circle).

applications where the localization and the identification of an object are required.

\section{Conclusion}

In this paper, we have shown that we can successfully localize a chipless tag using phase measurement. This localization can be realized without reducing the coding capacity since all resonators are used for identification and sensing. Moreover, we have shown that the accuracy of our proposed method is very good since distances are measured with a millimeter precision. Finally, tag localization has been done using a multilateration algorithm. Accuracy of our solution 


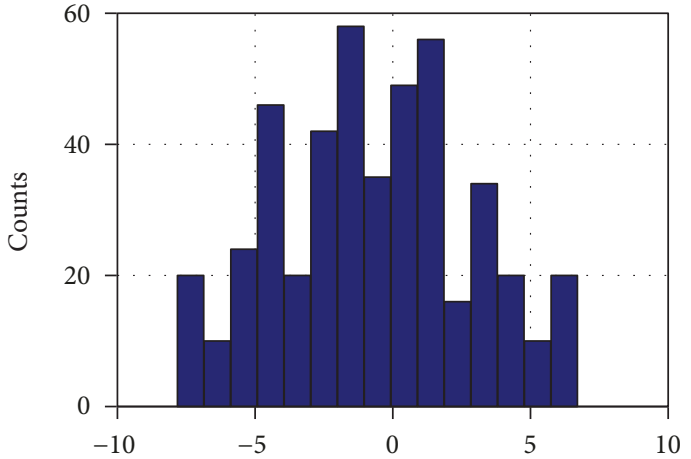

(a)

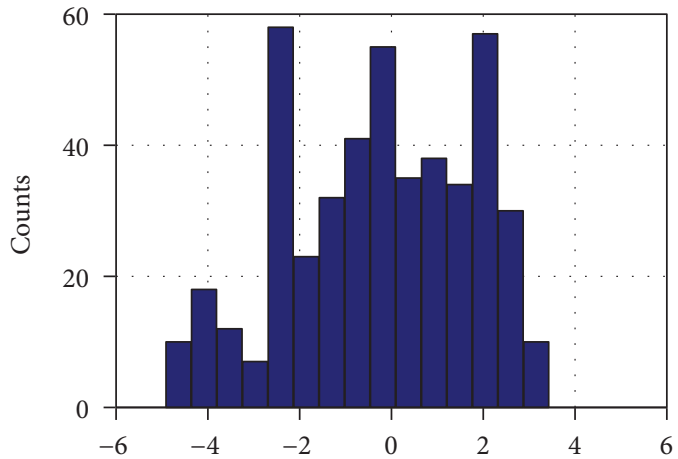

(b)

Figure 8: Localization error (a) along the $x$ and (b) the $y$ axes.

has been evaluated and is lower than $4 \mathrm{~mm}$. Finally, this method can easily be extended to determine $3 \mathrm{D}$ positioning and/or orientation.

\section{Data Availability}

The data used to support the findings of this study are included within supplementary materials.

\section{Conflicts of Interest}

The authors declare that there is no conflict of interest regarding the publication of this paper.

\section{Acknowledgments}

This project has received funding from the Institut Universitaire de France (IUF).

\section{References}

[1] A. Vena, E. Perret, and S. Tedjini, Chipless RFID Based on RF Encoding Particle: Realization, Coding and Reading System, ISTE; Elsevier, 2016.

[2] A. Vena, E. Perret, S. Tedjini et al., "Design of chipless RFID tags printed on paper by flexography," IEEE Transactions on Antennas and Propagation, vol. 61, no. 12, pp. 5868-5877, 2013.

[3] S. Hu, Y. Zhou, C. L. Law, and W. Dou, "Study of a uniplanar monopole antenna for passive chipless UWB-RFID localization system," IEEE Transactions on Antennas and Propagation, vol. 58, no. 2, pp. 271-278, 2010.

[4] R.-E.-A. Anee and N. C. Karmakar, "Chipless RFID tag localization," IEEE Transactions on Microwave Theory and Techniques, vol. 61, no. 11, pp. 4008-4017, 2013.

[5] R. Rezaiesarlak and M. Manteghi, "A space-frequency technique for chipless RFID tag localization," IEEE Transactions on Antennas and Propagation, vol. 62, no. 11, pp. 5790-5797, 2014.

[6] E. Perret, "Displacement sensor based on radar crosspolarization measurements," IEEE Transactions on Microwave Theory and Techniques, vol. 65, no. 3, pp. 955-966, 2017.

[7] N. Barbot and E. Perret, "Gesture recognition with the chipless RIFD technology," in 2017 XXXIInd General Assembly and
Scientific Symposium of the International Union of Radio Science (URSI GASS), pp. 1-3, Montreal, QC, Canada, 2017.

[8] E. Perret, Radio Frequency Identification and Sensors: From RFID to Chipless RFID, John Wiley \& Sons, Inc., Hoboken, NJ, USA, 2014.

[9] A. Vena, E. Perret, and S. Tedjni, "A depolarizing chipless RFID tag for robust detection and its FCC compliant UWB reading system," IEEE Transactions on Microwave Theory and Techniques, vol. 61, no. 8, pp. 2982-2994, 2013.

[10] M. A. Herráez, D. R. Burton, M. J. Lalor, and M. A. Gdeisat, "Fast two-dimensional phase-unwrapping algorithm based on sorting by reliability following a noncontinuous path," Applied Optics, vol. 41, no. 35, pp. 7437-7444, 2002.

[11] B. T. Fang, "Simple solutions for hyperbolic and related position fixes," IEEE Transactions on Aerospace and Electronic Systems, vol. 26, no. 5, pp. 748-753, 1990.

[12] D. E. Manolakis, "Efficient solution and performance analysis of 3-D position estimation by trilateration," IEEE Transactions on Aerospace and Electronic Systems, vol. 32, no. 4, pp. 12391248, 1996. 


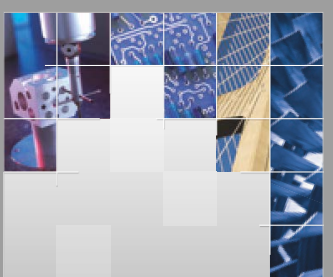

\section{Enfincering}
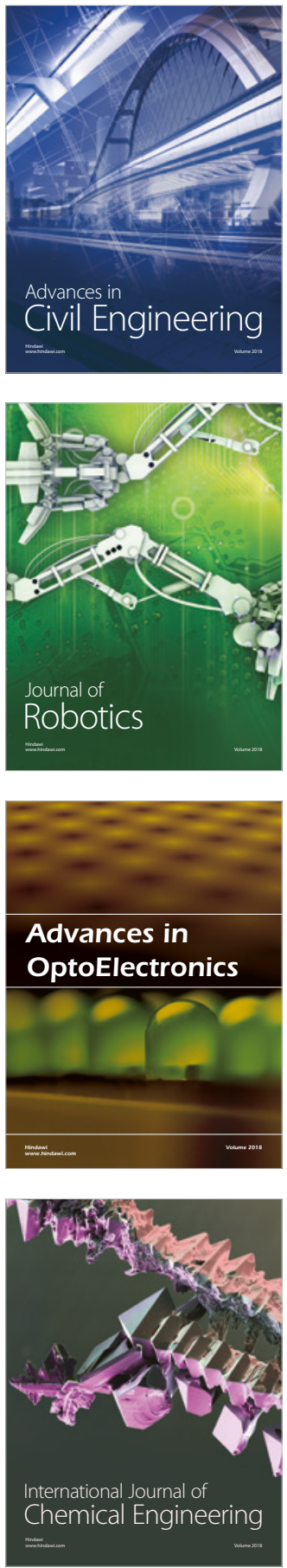

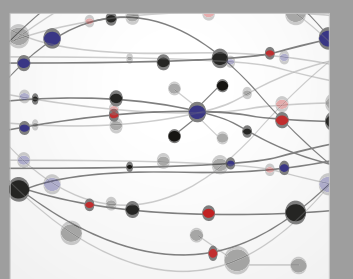

\section{Rotating \\ Machinery}

The Scientific World Journal

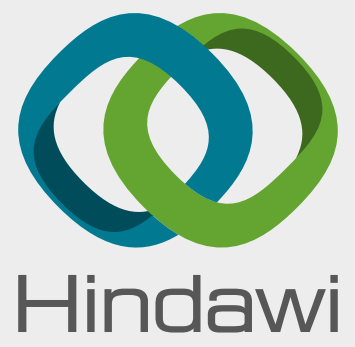

Submit your manuscripts at

www.hindawi.com
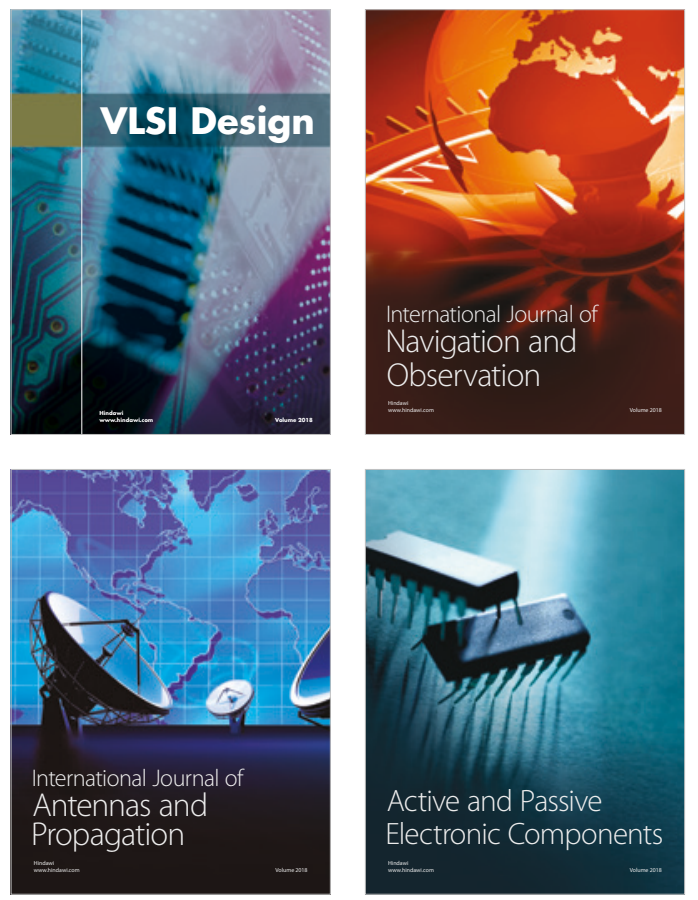
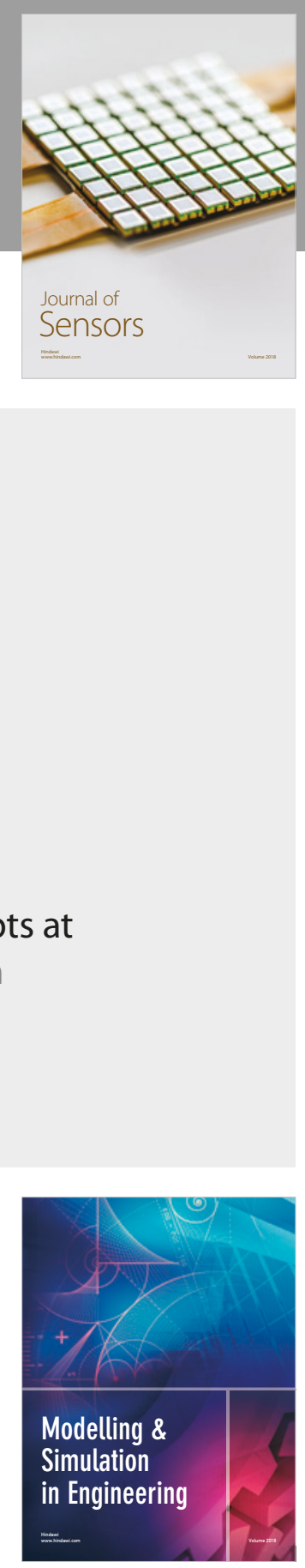

\section{Advances \\ Multimedia}
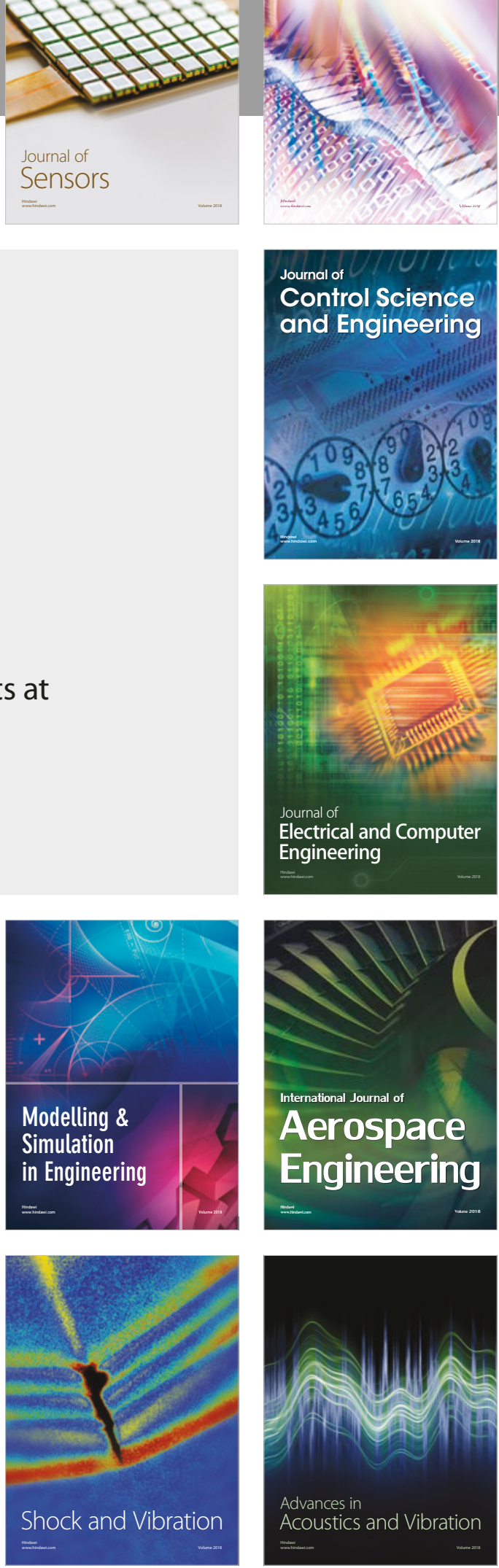